\title{
Pain coping skills training for African Americans with osteoarthritis study: baseline participant characteristics and comparison to prior studies
}

Kelli D. Allen ${ }^{1,2,3^{*}}$, Liubov Arbeeva ${ }^{1,2}$, Crystal W. Cené ${ }^{2}$, Cynthia J. Coffman ${ }^{3,4}$, Kimberlea F. Grimm $^{1,2}$, Erin Haley ${ }^{5}$, Francis J. Keefe ${ }^{6}$, Caroline T. Nagle ${ }^{2}$, Eugene Z. Oddone ${ }^{3,7}$, Tamara J. Somers ${ }^{6}$, Yashika Watkins ${ }^{8}$

and Lisa C. Campbell ${ }^{5}$

\begin{abstract}
Background: The Pain Coping Skills Training for African Americans with OsteoaRTthritis (STAART) trial is examining the effectiveness of a culturally enhanced pain coping skills training (CST) program for African Americans with osteoarthritis $(\mathrm{OA})$. This disparities-focused trial aimed to reach a population with greater symptom severity and risk factors for poor pain-related outcomes than previous studies. This paper compares characteristics of STAART participants with prior studies of CST or cognitive behavioral therapy (CBT)-informed training in pain coping strategies for OA.

Methods: A literature search identified 10 prior trials of pain CST or CBT-informed pain coping training among individuals with OA. We descriptively compared characteristics of STAART participants with other studies, in 3 domains of the National Institutes of Minority Health and Health Disparities' Research Framework: Sociocultural Environment (e.g., age, education, marital status), Biological Vulnerability and Mechanisms (e.g, pain and function, body mass index), and Health Behaviors and Coping (e.g., pain catastrophizing). Means and standard deviations (SDs) or proportions were calculated for STAART participants and extracted from published manuscripts for comparator studies.

Results: The mean age of STAART participants, 59 years (SD =10.3), was lower than 9 of 10 comparator studies; the proportion of individuals with some education beyond high school, $75 \%$, was comparable to comparator studies (61-86\%); and the proportion of individuals who are married or living with a partner, 42\%, was lower than comparator studies (62-66\%). Comparator studies had less than about 1/3 African American participants. Mean scores on the Western Ontario and McMaster Universities Osteoarthritis Index pain and function scales were higher (worse) for STAART participants than for other studies, and mean body mass index of STAART participants, $35.2 \mathrm{~kg} / \mathrm{m}^{2}(\mathrm{SD}=8.2)$, was higher than all other studies $\left(30-34 \mathrm{~kg} / \mathrm{m}^{2}\right)$. STAART participants' mean score on the Pain Catastrophizing scale, 19.8 (SD = 12.3), was higher (worse) than other studies reporting this measure (7-17).

\footnotetext{
* Correspondence: kdallen@email.unc.edu

${ }^{1}$ Thurston Arthritis Research Center, University of North Carolina at Chape Hill, 3300 Thurston Bldg., CB\# 7280, Chapel Hill, NC 27599-7280, USA ${ }^{2}$ Department of Medicine, University of North Carolina at Chapel Hill, 125 MacNider Hall CB\# 7005, Chapel Hill, NC 27599, USA

Full list of author information is available at the end of the article
}

(c) The Author(s). 2018 Open Access This article is distributed under the terms of the Creative Commons Attribution 4.0 International License (http://creativecommons.org/licenses/by/4.0/), which permits unrestricted use, distribution, and reproduction in any medium, provided you give appropriate credit to the original author(s) and the source, provide a link to the Creative Commons license, and indicate if changes were made. The Creative Commons Public Domain Dedication waiver (http://creativecommons.org/publicdomain/zero/1.0/) applies to the data made available in this article, unless otherwise stated. 
(Continued from previous page)

Conclusions: Compared with prior studies with predominantly white samples, STAART participants have worse pain and function and more risk factors for negative pain-related outcomes across several domains. Given STAART participants' high mean pain catastrophizing scores, this sample may particularly benefit from the CST intervention approach.

Trial registration: NCT02560922

Keywords: Osteoarthritis, Knee, Hip, Pain coping skills training, Health disparities

\section{Background}

African Americans bear a disproportionate burden of chronic pain conditions, including osteoarthritis (OA). Compared with Caucasians, African Americans not only have a higher prevalence of OA, but also more severe pain, functional limitations, and other negative outcomes [1-6]. Because of these well-documented racial differences, the Institute of Medicine has identified interventions to reduce disparities in OA and other musculoskeletal diseases among its top 25 (highest tier) priority topics for comparative effectiveness research [7]. In alignment with this priority, the Pain Coping Skills Training for African Americans with OsteoaRTthritis (STAART) study is evaluating the effectiveness of a culturally enhanced pain coping skills training (CST) program for African Americans with OA [8]. There were several factors underlying the choice of a pain CST intervention to address racial disparities in OA-related pain and other outcomes. First, when compared with Caucasians, African Americans with chronic pain conditions report greater levels of pain catastrophizing (i.e., the tendency to focus on and magnify pain sensations and to feel helpless in the face of pain [9-13]), lower perceived ability to cope with and control pain [14], and greater maladaptive coping strategies (i.e., emotion-focused or external coping strategies) $[4,10,14-16]$. These coping-related characteristics have been associated with worse pain, function, and depressive symptoms [17-19]. Second, previous studies indicated that pain coping and other psychological factors are key factors underlying racial differences in OA-related pain [3, 4]. Third, pain CST interventions have been shown to enhance and improve coping strategies, OA-related pain and other outcomes [20-25]. However, there has been very limited study of pain CST interventions among African Americans with OA or other musculoskeletal conditions. This is important because of evidence that behavioral and psychological interventions are most effective when they are adapted to meet the needs and expectations of minority populations [26].

Based on prior studies of racial differences in pain, coping, and social determinants of health [3, 11-16, 27, 28], we expected that baseline characteristics of the STAART study participants, who are all African American, would reflect a worse risk profile than those of participants in prior studies of pain CST or other cognitive behavioral therapy (CBT)-informed training in pain coping strategies. Therefore, the objective of this analysis was to descriptively compare characteristics of STAART study participants with prior studies of pain CST or CBT-informed pain coping strategies among individuals with OA. In particular, we focused on Individual Level Sociocultural Environment, Biological and Behavioral domains, within the National Institute on Minority Health and Health Disparities (NIMHD) Research Framework [29] as these are of highest relevance to this intervention and population. This Framework also has domains at the Interpersonal Level (e.g., family functioning, patient-clinician relationship), Community Level (e.g. community resources, availability of health services), and Societal Level (e.g., policies and laws); although some items in these domains are also relevant to this intervention and patient group, variables within these domains were not assessed in STAART.

\section{Methods}

\section{Overview of STAART study}

The STAART study, described in detail previously [8], is a randomized controlled trial of 248 African Americans with symptomatic hip or knee OA. The STAART study enrolled only African Americans (vs inclusion of other racial groups) so that in-depth efforts could be focused on this demographic group with a high risk for poorer OA and pain-related outcomes. STAART participants are equally allocated to pain CST and wait list control groups. The pain CST intervention involved 11 phone-based sessions, delivered approximately weekly and based on previous pain CST programs [20, 22, 23, 25, 30]. Participants in the wait list group received only their usual care for OA, with no study intervention offered until after completion of final follow-up assessments. All measures for these analyses were collected from patients prior to their randomization to treatment conditions. This study was approved by the Institutional Review Boards of the University of North Carolina (UNC), the Durham VA Healthcare System (VA), Duke University Medical Center and East Carolina University.

\section{STAART participants and recruitment methods}

Participants were recruited from the UNC Healthcare System and the Durham VA; 124 participants were 
enrolled from each site. Study inclusion were 1) Diagnosis of knee or hip OA, verified by self-reported diagnosis from a medical professional, including items based on the American College of Rheumatology clinical criteria for knee or hip OA, 2) Self-report of pain, aching, or stiffness in one or both knees or hips on most days of the week, 3) Patient of the UNC Health Care System or Durham VAMC. Exclusion criteria have been described previously [8] and generally include other pain-related conditions that confound study outcomes or health conditions that would prevent participation in the intervention (e.g., severe hearing loss since this was a phone-based intervention).

Three general methods of recruitment were used. First, potentially eligible patients were identified from $\mathrm{UNC}$ and VA medical records, based on OA diagnosis codes; these patients were mailed letters inviting participation, followed by a telephone call. Second, advertisements were posted at study sites and surrounding communities, inviting patients to self-refer to the study. Third, health care providers at study sites could refer patients to the study team directly, with patients' permission, or give study brochures to patients. We used an enhanced informed consent process that included education about the research process, participant bill of rights, and perspectives from African Americans who have participated in research [31].

\section{Measures}

The following measures, representing three Individual Level domains from the NIMHD Research Framework, were assessed in-person at baseline by a trained research assistant. Some of these measures are reported for the STAART study sample only (Table 1) because they were not available for any comparator studies, but they represent key constructs related to health disparities and the NIMHD Framework.

\section{Sociodemographics (individual level sociocultural environment domain)}

Age Participant age was based on date of birth from the electronic medical record and confirmed through self-report.

Sex Participant sex was based on the electronic medical record and confirmed through self-report.

Ethnicity Participants self-reported whether they were of Hispanic / Latino descent or not.

Education Participants selected from eight options ranging from grade school/junior high to post graduate work or graduate degree. For these analyses we grouped individuals as having "above high school education" or not, as this was the most common categorization that could be ascertained from comparator studies.

Work status Participants selected from seven options regarding work status, and for these analyses individuals were grouped as either working or not at the time of the study.

Household financial status Participants selected from four options regarding their household's financial situation and were grouped as either "live comfortably" or "meet basic expenses with a little left over for extras" vs. "just meet basic expenses" or "don't even have enough to meet basic expenses."

Marital status Participants selected from five options regarding current marital status and for these analyses were grouped as being married / living with a partner as married or not currently married at the time of the study.

Religiosity This measure was included in STAART because of its cultural relevance in the African American community. The Duke University Religion Index (DUREL) is a five-item measure of religious beliefs and experience [32]. The index consists of 3 subscales, recording the frequency of attendance at religious services (subscale 1; range 1-6), the frequency of private religious activities (subscale 2; range 1-6) and intrinsic religiosity (subscale 3; range 3-15). Higher scores represent more religious activities or religiosity.

\section{Biological vulnerability and mechanisms (individual level biological domain)}

Pain and function - Western Ontario and McMaster Universities Osteoarthritis Index (WOMAC) The WOMAC is a measure of lower extremity pain (5 items), stiffness (2 items), and function (17 items) [33, 34]. All items were rated on a Likert scale of 0 (no symptoms) to 4 (extreme symptoms), with a total range of 0-96 and higher scores indicating worse symptoms. Pain and function subscales are also separately reported. Some other studies used the Visual Analog Scale (VAS) version of the WOMAC, which includes the same items but each measured on a $100 \mathrm{~mm}$ VAS. For this version, each subscale score ranges from 0 to 100 , with higher scores indicating worse symptoms or function. To facilitate comparison of STAART with studies using the WOMAC VAS version, we also transformed pain and function domains to a $0-100$ scale, which has been done in prior studies $[35,36]$.

Arthritis impact measurement scales (AIMS) The AIMS was not collected in the STAART study. However, 
Table 1 Characteristics of STAART Participants, Overall and By Site

\begin{tabular}{|c|c|c|c|}
\hline Characteristic & $\begin{array}{l}\text { Total Sample } \\
\text { Mean } \pm \text { SD or N(\%) }\end{array}$ & $\begin{array}{l}\text { DVAHCS } \\
\text { Mean } \pm \text { SD or } \mathrm{N}(\%)\end{array}$ & $\begin{array}{l}\text { UNC } \\
\text { Mean } \pm \text { SD or } \mathrm{N}(\%)\end{array}$ \\
\hline \multicolumn{4}{|l|}{ Sociocultural Environment } \\
\hline Age & $59.0 \pm 10.3$ & $57.8 \pm 10.0$ & $60.2 \pm 10.5$ \\
\hline Female & $122(49.2 \%)$ & $26(21.0 \%)$ & $96(77.4 \%)$ \\
\hline Hispanic & $7(2.9 \%)$ & $2(1.6 \%)$ & $5(4.3 \%)$ \\
\hline Some education above high school & $187(75.4 \%)$ & $101(81.5 \%)$ & $86(69.4 \%)$ \\
\hline Working & $86(34.7 \%)$ & 47 (37.9\%) & $39(31.5 \%)$ \\
\hline Married or living with partner & $103(41.5 \%)$ & $63(50.8 \%)$ & $40(32.3 \%)$ \\
\hline Low Perceived Income ${ }^{a}$ & $83(33.6 \%)$ & 39 (31.7\%) & $44(35.5 \%)$ \\
\hline \multicolumn{4}{|l|}{ Duke University Religion Index } \\
\hline Attendance at Religious Activities & $4.4 \pm 1.4$ & $4.1 \pm 1.5$ & $4.8 \pm 1.2$ \\
\hline Private Religious Activities & $4.0 \pm 1.6$ & $3.8 \pm 1.6$ & $4.2 \pm 1.5$ \\
\hline Intrinsic Religiosity & $13.1 \pm 2.5$ & $13.1 \pm 2.4$ & $13.2 \pm 2.6$ \\
\hline \multicolumn{4}{|l|}{ Biological Vulnerability and Mechanisms } \\
\hline WOMAC Total ${ }^{\mathrm{b}}$ & $53.0 \pm 17.8$ & $56.0 \pm 16.6$ & $49.9 \pm 18.5$ \\
\hline WOMAC Pain & $11.0 \pm 3.9$ & $11.6 \pm 3.8$ & $10.4 \pm 3.9$ \\
\hline WOMAC Function & $37.0 \pm 13.2$ & $39.1 \pm 12.4$ & $34.9 \pm 13.7$ \\
\hline PROMIS Pain Interference Score & $63.8 \pm 6.9$ & $64.0 \pm 6.3$ & $63.5 \pm 7.5$ \\
\hline Short Form-12 - Mental & $50.7 \pm 11.1$ & $49.8 \pm 11.6$ & $51.5 \pm 10.7$ \\
\hline Short Form-12 - Physical & $33.1 \pm 9.1$ & $33.1 \pm 8.4$ & $33.1 \pm 9.7$ \\
\hline Duration of Arthritis Symptoms & $13.1 \pm 10.0$ & $14.6 \pm 10.6$ & $11.5 \pm 9.1$ \\
\hline Number of Self-Reported Comorbidities & $8.5 \pm 3.9$ & $8.2 \pm 3.5$ & $8.8 \pm 4.3$ \\
\hline Body Mass Index (kg/m²) & $35.2 \pm 8.2$ & $33.1 \pm 6.5$ & $37.5 \pm 9.1$ \\
\hline \multicolumn{4}{|l|}{ Health Behaviors \& Coping Strategies } \\
\hline CSQ - Total Coping Attempts & $93.9 \pm 36.6$ & $91.9 \pm 39.7$ & $95.8 \pm 33.3$ \\
\hline CSQ - Diverting Attention & $13.9 \pm 8.4$ & $12.9 \pm 8.6$ & $14.8 \pm 8.2$ \\
\hline CSQ - Ignoring Sensations & $14.1 \pm 8.7$ & $15.0 \pm 9.6$ & $13.2 \pm 7.7$ \\
\hline CSQ - Coping Self-Statements & $23.6 \pm 7.6$ & $23.4 \pm 8.2$ & $23.7 \pm 6.8$ \\
\hline CSQ - Reinterpreting Pain Sensations & $8.9 \pm 8.4$ & $9.7 \pm 8.8$ & $8.1 \pm 8.0$ \\
\hline CSQ - Praying, Hoping & $20.8 \pm 8.0$ & $19.8 \pm 8.0$ & $21.9 \pm 7.9$ \\
\hline CSQ - Increasing Behavioral Activities & $12.5 \pm 7.1$ & $11.0 \pm 7.0$ & $14.0 \pm 6.9$ \\
\hline CSQ - Catastrophizing & $11.4 \pm 7.6$ & $11.1 \pm 7.3$ & $11.7 \pm 7.8$ \\
\hline Pain Catastrophizing Scale & $19.8 \pm 12.3$ & $20.4 \pm 12.4$ & $19.2 \pm 12.1$ \\
\hline PHQ-8 & $6.2 \pm 5.3$ & $6.6 \pm 5.3$ & $5.7 \pm 5.3$ \\
\hline Arthritis Self-Efficacy Scale & $5.9 \pm 2.0$ & $5.6 \pm 2.1$ & $6.1 \pm 1.8$ \\
\hline Self-Efficacy for Pain Communication & $78.7 \pm 22.0$ & $77.4 \pm 22.9$ & $80.1 \pm 21.1$ \\
\hline Brief Fear of Movement Scale & $14.8(3.5)$ & $14.4(3.9)$ & $15.2(3.1)$ \\
\hline
\end{tabular}

DVAHCS Durham VA Health Care System, UNC University of North Carolina, WOMAC Western Ontario and MCMaster Universities Osteoarthritis Index, CSQ coping strategies questionnaire, $P H Q$ patient health questionnaire

a Self-report of "just meet basic expenses" or "don't even have enough to meet basic expenses."

${ }^{b}$ Likert scale version of WOMAC

it is a common measure in other OA studies, and therefore we present it for comparator studies when available. Although this does not allow a direct comparison to WOMAC, the AIMS scale provides a general description of the symptom severity of participants in comparator studies. Comparator studies used both the original AIMS, the AIMS2 and the AIMS2 Short Form (AIMS2-SF). The original AIMS includes 45 items across the domains of pain, physical disability and psychological disability [37]; the latter 2 are reported here because of their similarity to 
WOMAC domains. Each AIMS domain has a score range of $0-10$, with higher scores indicating greater pain or disability. The AIMS2 is an expanded version with 78 items, and the AIMS2-SF has 26 items [38, 39]. AIMS2 domains also have score ranges of $0-10$, with higher scores indicating greater pain or disability.

PROMIS pain interference instrument (short form) The PROMIS Pain Interference (Short Form 6a) instrument measures the self-reported consequences of pain across aspects of life including social, cognitive, emotional, physical and recreational activities; this instrument refers to the past 7 days [40] This validated scale has five response options, with scores ranging from one to five; items are summed and re-scaled as a t-score with mean of 50 and standard deviation of 10 .

Health-related quality of life (HRQoL) - Short form 12 (SF-12) The Short-Form-12 (SF-12) is a validated measure that covers domains of general health, physical health, work and activity limitations, and emotional health [41]. Physical and Mental Health Composite Scores (PCS \& MCS) are computed using the scores of 12 questions and range from 0 to 100 , with higher scores indicating better health.

Duration of arthritis symptoms Participants self-reported the number of years they have been experiencing knee and / or hip arthritis symptoms (joint pain, stiffness, or limited movement).

Comorbid illnesses The Self-Administered Comorbidity Questionnaire asks participants to indicate whether or not they have each of 13 physical and psychological health conditions. Participants can also list up to 3 additional conditions. The score range is $0-16$ [42].

Body mass index (BMI) BMI was calculated from measured height and weight at baseline.

\section{Health behaviors \& coping strategies (individual level behavioral domain)}

Coping strategies questionnaire (CSQ) The CSQ is the most commonly used measure of coping among individuals with chronic pain, and its measurement properties have been confirmed in patients with a variety of pain-related conditions [43, 44]. This scale includes 48 items that assess 6 cognitive domains (Catastrophizing, Diverting Attention, Ignoring Sensations, Coping Self-Statements, Reinterpreting Pain Sensations, Praying-Hoping) and 1 behavioral domain (Increasing Behavioral Activities). Each domain includes 6 items, and participants rate the frequency of their use of specific coping strategies on a 7-point Likert scale from 0 ("Never do that") to 6 ("Always do that"). From the CSQ, we calculated a Coping Attempts Score, which sums all domains other than Catastrophizing. This score was reported because it could be compared to other previous studies $[45,46]$, and because the factor structure for this score has been replicated in prior research [47-49]. We also separately report scores for the Catastrophizing subscale.

Pain catastrophizing scale (PCS) The PCS is a widely used measure of catastrophic thinking related to pain [50]. This 13-item instrument asks participants to reflect on past painful experiences and to indicate the degree to which they experienced each of the thoughts or feelings when experiencing pain, with each item scored from 0 (not at all) to 4 (all the time). The PCS includes 3 subscales - rumination, magnification, and helplessness. Scores for all items are summed and total scores range from 0 to 52 with a higher score indicating a higher level of catastrophizing.

Depressive symptoms - Patient health questionnaire 8 (PHQ-8) The PHQ-8 is an eight-item survey that consists of items corresponding to depression criteria listed in the Diagnostic and Statistics Manual Fourth Edition (DSM-IV) [51]. Each of the eight questions is scored as 0 (not at all) to 3 (nearly every day), so that total scores range from 0 to 24 .

Arthritis self efficacy scale This scale includes 8 items asking respondents how certain they are that they can perform specific activities or tasks [52]. Items are scored on a Likert Scale $(1=$ very uncertain to $10=$ very certain); the total score represents a mean of the 8-items, with a range of 1-10. Because of challenges with comparing scores across different versions of this scale, we only included comparator studies that used the 8-item version.

Self-efficacy for pain communication scale - Patient version [53] This 7-item instrument assesses patient's level of confidence in communicating their pain to a "significant other" and receiving understanding and a helpful response. Items are rated on a scale from 10 ("very uncertain") to 100 ("very certain").

Brief fear of movement scale The Brief Fear of Movement Scale is a six item scale for assessing fear of movement in OA [54]. All items are measured on a 4-point scale from "strongly agree" to "strongly disagree." The total score ranges from 6 to 24, with higher scores indicating more fear of movement. 


\section{Comparator studies}

We aimed to identify prior studies of pain CST and CBT-informed pain coping strategies among individuals with OA (regardless of racial composition), since those are of highest relevance to the STAART study. To identify comparator studies, we performed a literature search (using Pubmed) with search terms of (osteoarthritis) AND (CST OR CBT). We included clinical trials meeting these criteria from any country, resulting in 10 studies. We also compared our identified studies to a recent systematic review of behavioral intervention for OA and found no additional studies to include. For each of study we extracted relevant baseline participant characteristics for comparison to STAART. When participant characteristics were presented only by treatment arm, we contacted authors to request characteristics for the full study sample for simplicity of comparisons. When these were not available we presented characteristics by treatment arm. We compared characteristics between STAART participants and other studies descriptively. Because of the relatively small number of studies and because not all studies assessed all measures of interest, we did not conduct statistical comparisons.

The following are summaries of the studies we identified and included in this comparison. Additional details on participant inclusion criteria and recruitment methods are reported in Additional file 1:

Effectiveness of an Internet-Delivered Exercise and Pain-Coping Skills Training Intervention for Persons With Chronic Knee Pain: A Randomized Trial (Bennell et al., 2017) [45].

- Participants: 148 patients with knee pain.

- Intervention: online educational materials, an interactive, automated 8-module pain CST program (PainCOACH), and seven Skype sessions with a physiotherapist for 12 weeks, focusing on a home exercise program.

- Comparator group: online educational materials only.

Physical Therapist-Delivered Pain Coping Skills Training and Exercise for Knee Osteoarthritis: Randomized Controlled Trial (Bennell et al., 2016) $[55,56]$.

- Participants: 222 patients with symptomatic knee OA.

- Interventions: pain CST only, exercise only or pain CST/exercise combined. All groups attended 10 individual sessions with a physical therapist for 12 weeks, plus a home program.

Automated Internet-based pain coping skills training to manage osteoarthritis pain: a randomized controlled trial (Rini et al., 2015) [57].

- Participants: 113 participants with hip or knee OA
- Interventions: Internet-based PCST (PainCOACH), eight modules in a self-directed manner at a rate of one per week

- Comparator group: assessment-only control group

Nurse practitioners can effectively deliver pain coping skills training to osteoarthritis patients with chronic pain: A randomized, controlled trial (Broderick et al, 2014) [58].

- Participants: 256 participants with symptomatic knee or hip OA

- Intervention: 10 individual weekly sessions of pain CST

- Comparator group: usual care

Effectiveness of a cognitive-behavioural group intervention for knee osteoarthritis pain: a randomized controlled trial (Helminen et al., 2014) [59].

- Participants: 111 patients with symptomatic knee OA

- Intervention: CBT program for pain management, delivered in 6 weekly group sessions led by both a psychologist and a physiotherapist

- Comparator group: regular general practitioner care only

Cognitive-behavioral treatment for comorbid insomnia and osteoarthritis pain in primary care: the lifestyles randomized controlled trial (Vitiello et al., 2013) [60].

- Participants: 367 individuals with symptomatic OA and insomnia,

- Interventions: CBT for pain and insomnia, CBT for pain or education. CBT interventions were delivered in groups at primary care clinics and consisted of 6 weekly 90-min sessions.

- Comparator group: usual care

Pain coping skills training and lifestyle behavioral weight management in patients with knee osteoarthritis: a randomized controlled study (Somers et al., 2012) [25].

- Participants: 232 individuals with symptomatic knee OA

- Interventions: pain CST plus lifestyle behavioral weight management (BWM), pain CST only, BMW only. Pain CST only and BWM only interventions had 12 weekly 60-min sessions, followed by biweekly 60-min sessions for 12 weeks. The BWM only group also had three weekly supervised sessions weekly for the first 12 weeks. The pain CST + BWM group had 12 weekly 120 min sessions, in addition to 3 weekly supervised exercise sessions, followed by bi-weekly 120-min sessions for 12 weeks.

- Comparator group: standard care 
Clinical effectiveness of a rehabilitation program integrating exercise, self-management, and active coping strategies for chronic knee pain: a cluster randomized trial (Hurley et al., 2007) [61].

- Participants: 418 individuals with knee pain.

- Interventions: individual rehabilitation, group rehabilitation (8 patients per group). Both individual and group rehabilitation involved 12 sessions (twice weekly for 6 weeks), supervised by a physiotherapist. Content included instruction in pain coping and self-management, as well as an individualized progressive exercise program.

- Comparator group: usual care

Spouse-assisted coping skills training in the management of osteoarthritic knee pain (Keefe et al., 1996) [46].

- Participants: 88 married persons with knee OA

- Interventions: spouse assisted pain CST, conventional pain CST with no spouse involvement arthritis education-spousal support control. Participants in all three interventions met in groups of 4 to 6 patients (or couples) for 10 weekly, 2-h group sessions.

Pain coping skills training in the management of osteoarthritic knee pain: A comparative study (Keefe et al., 1990) $[20,62]$.

- Participants: 99 patients with knee OA

- Interventions: pain CST, arthritis education. Both interventions met in small groups (6 to 9 patients) for 10 weekly 90 -min sessions.

- Comparator: standard care control

\section{Results}

At both STAART study sites (UNC, Durham VA), 124 participants were enrolled. At UNC, 381 participants refused and 123 were ineligible; at the Durham VA, 632 participants refused and 77 were ineligible. At UNC, the mean ages for consented, refused, and ineligible patients, respectively, were: 60.2 (standard deviation $(\mathrm{SD})=10.5$ ), 64.0 ( $\mathrm{SD}=12.9)$, and $60.1(\mathrm{SD}=12.7)$; the proportions of females among those consented, refused, and ineligible, respectively, were: 77,69 and $70 \%$. At the VA, the mean ages for consented, refused, and ineligible patients, respectively were: $57.8(\mathrm{SD}=10.0), 59.9(\mathrm{SD}=11.3)$, and $61.2(\mathrm{SD}=11.1)$; the proportions of females among those consented, refused, and ineligible, respectively, were: 21.0, 11.7 and 18.2\%. Characteristics of consented STAART participants, overall and by site, are shown in Table 1. Table 2 compares Sociocultural Environment, Biological Vulnerability and Mechanisms, and Health
Behaviors and Coping variables for STAART participants and comparator studies; this table includes variables for which there was at least one comparator study that included the measure.

\section{Sociocultural environment \\ Age}

The mean age of STAART participants was 59 years ( $S D=10.3)$, with a slightly lower age for VA participants than UNC participants. This mean age was slightly lower than other studies except for Somers et al. [25].

Sex

The proportion of females in the STAART study was $49 \%$, which is lower than in other studies (range: 56$81 \%$ ); among VA participants, only $21 \%$ were female, which reflects the high proportion of males in the VA.

\section{Race and ethnicity}

All STAART participants self-identified during screening as being black or African American, per study eligibility requirements. Two other studies included about $1 / 3$ African Americans [25, 57], but the rest 13\% or fewer (though several studies did not report information on race). Among STAART participants, $2.9 \%$ also self-identified as being of Hispanic or Latino ethnicity. Only Rini et al. reported ethnicity information for the sample, with $11 \%$ being of Hispanic or Latino ethnicity [57].

\section{Education}

Among STAART participants, 75\% reported some education above high school, with the proportion being higher among VA than UNC participants. Proportions of participants with education above high school ranged from 61 to $86 \%$ in comparator studies.

\section{Work status}

Thirty-four percent of STAART participants reported they were currently working. Proportions of working participants ranged widely among other studies, from 21 to $57 \%$.

\section{Household financial status}

Among STAART participants, about $1 / 3$ reported that they could "just meet basic expenses" or "don't have enough to meet basic expenses." We did not identify any comparator studies that measured financial or income status in a manner that could be directly compared with the measure we collected for STAART participants.

\section{Marital status}

Forty-two percent of STAART participants were married or living with a partner as married, with a substantially 


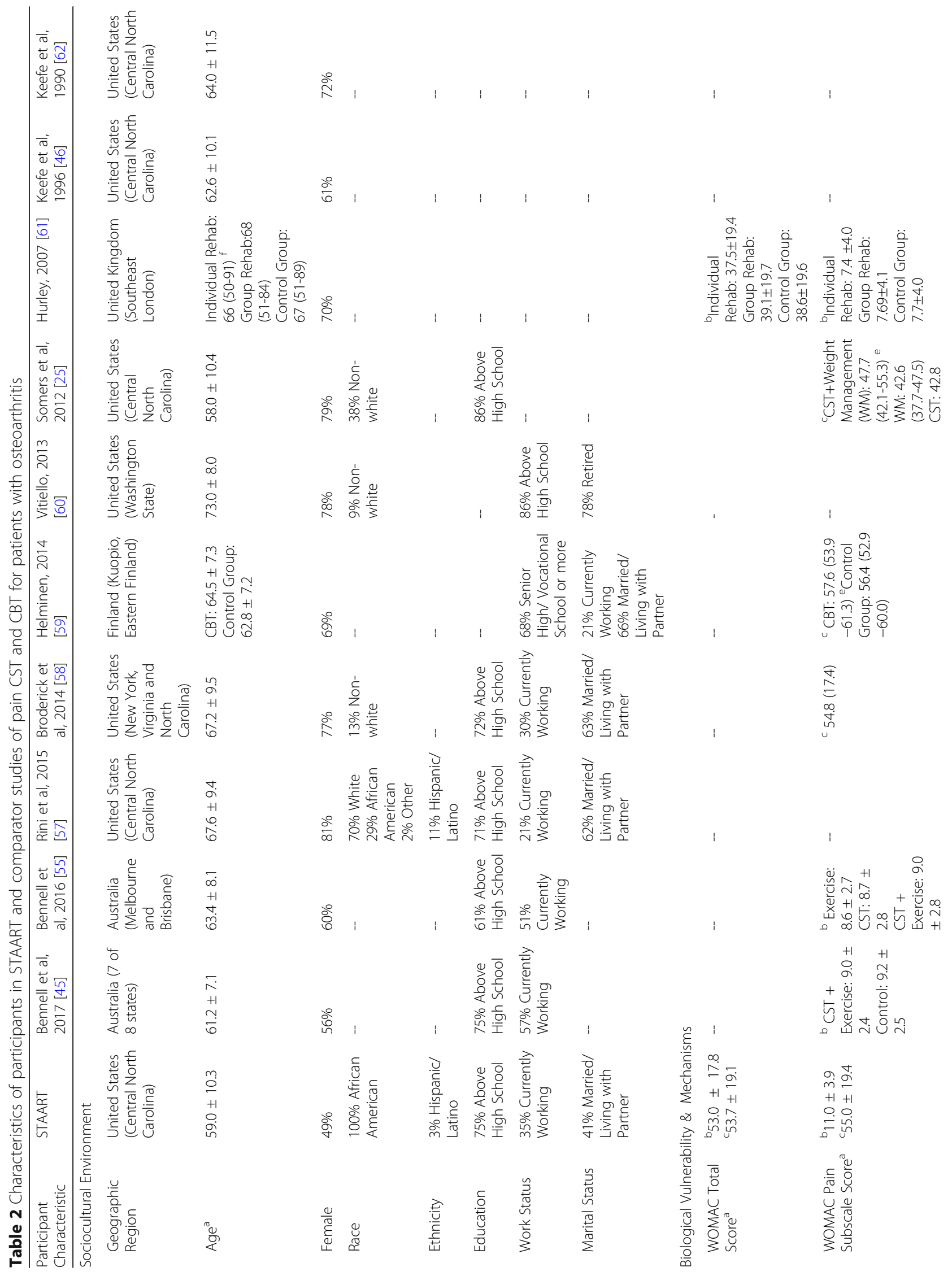




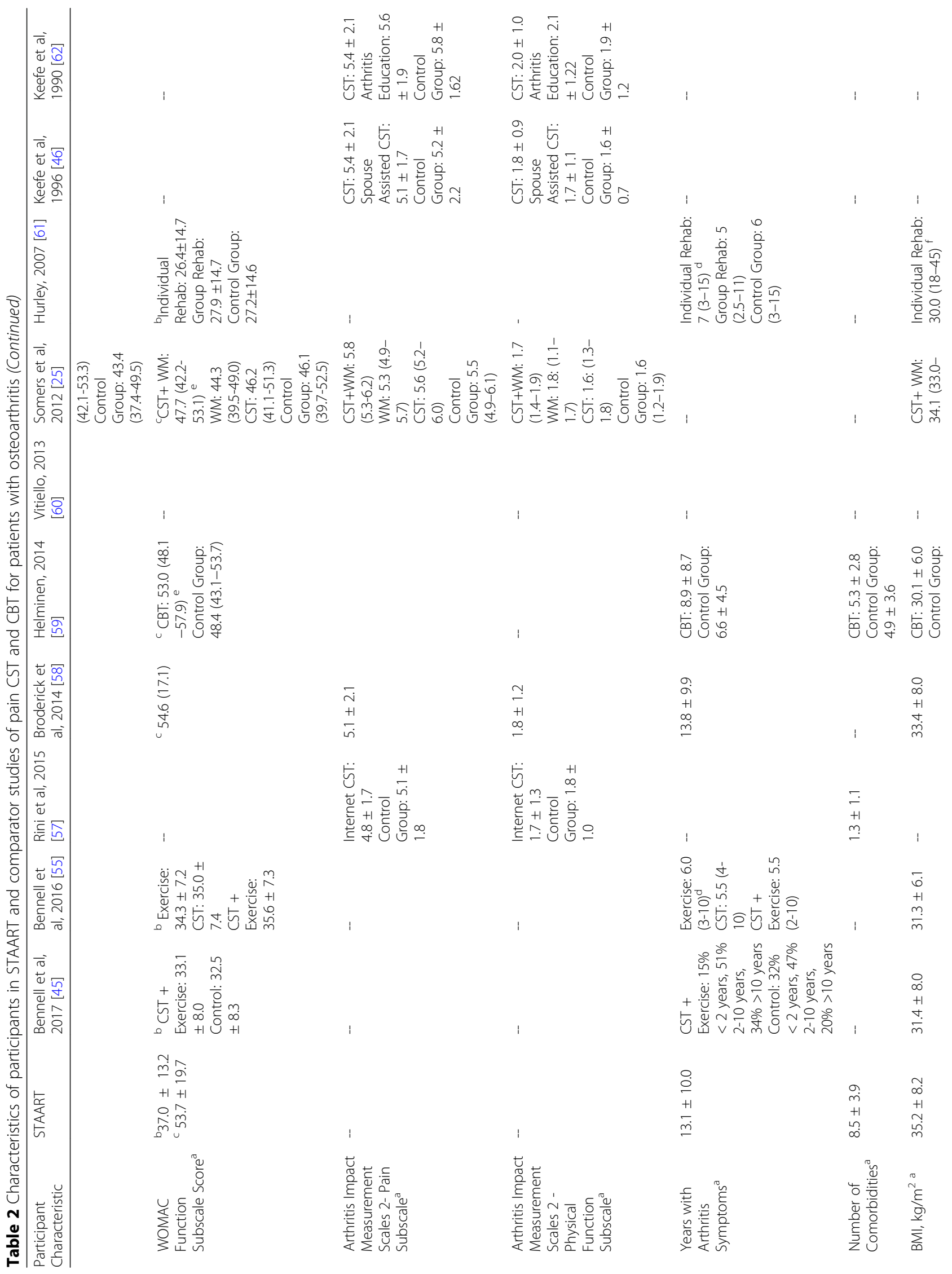




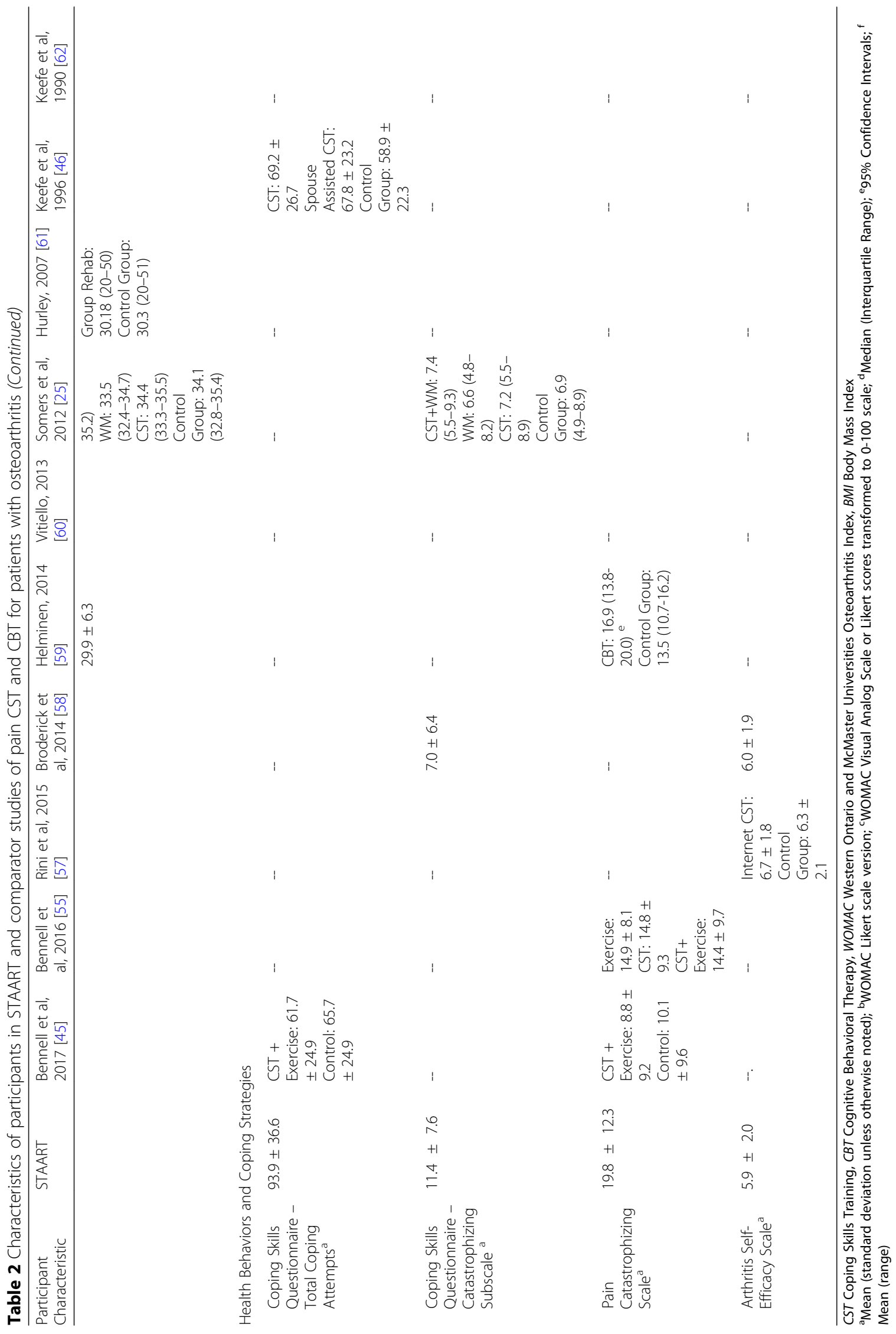


higher proportion for VA than UNC (51\% vs. $32 \%)$. In other studies, the proportions of married participants were all higher, ranging from 62 to $66 \%$, though a number of studies did not report marital status. This comparison reflects a potentially greater need or risk for STAART participants.

\section{Religiosity}

Among STAART participants, mean scores on the DUREL were relatively high for all domains, including attendance at religious services, private religious activities and intrinsic religiosity. We did not identify any comparator studies that measured this construct.

\section{Biological vulnerability and mechanisms \\ Pain and function - WOMAC}

The mean total WOMAC score (Likert version) among STAART participants was $53.0(\mathrm{SD}=17.8)$, which indicates moderate to severe symptoms. WOMAC scores were somewhat worse for the VA group compared to the UNC group. One comparator study reported total WOMAC scores (Likert version) ranging from 38 to 39 [61]; this comparison indicates greater symptom severity among STAART participants. The mean WOMAC pain subscale score (Likert version) among STAART participants was 11.0 $(\mathrm{SD}=3.9)$. Among three comparator studies that reported WOMAC pain scores on the Likert scale, mean values were all lower than for STAART participants (7.7-8.6); this comparison indicates greater pain among STAART participants [45, 55, 61]. When converted to a 0-100 scale, the mean WOMAC pain score among STAART participants was $55.0(\mathrm{SD}=19.4)$; this score was worse than [25] or comparable to [59] comparator studies that used the WOMAC VAS version. The mean WOMAC function subscale score (Likert version) among STAART participants was $37.0(\mathrm{SD}=13.2)$. Among three comparator studies that reported WOMAC function scores on the Likert scale, mean values were all lower (26-33); this comparison reflects poorer function among STAART participants $[45,55,61]$. When converted to a 0-100 scale, the mean WOMAC function score among STAART participants was $53.7(\mathrm{SD}=19.7)$; this score was worse than [25] or comparable to [58, 59] other studies that measured the WOMAC VAS version.

\section{Pain - AIMS}

Several studies included AIMS or AIMS2 pain and function scores. Among these, AIMS pain scores ranged from 5.1-5.8 and AIMS2 pain scores ranged from 4.8-5.1; these scores represent modest levels of pain (scale range of 0 10). AIMS function scores ranged from 1.6-2.0 and AIMS2 function scores ranged from 1.7-1.8; these scores represent relatively low levels of functional limitations, potentially reflecting that these samples were less limited than participants in the STAART study.

\section{Pain interference - PROMIS}

Among STAART study participants, the mean score was $63.8(\mathrm{SD}=6.9)$. This score indicates that the mean level of pain interference for STAART participants was a little over one standard deviation greater than the average of the general population. We did not identify any comparator studies that included this measure.

\section{HRQoL - SF-12}

The mean SF-12 PCS score for STAART participants was 33.1; this is lower than the average score for US men and women aged 60-69 (45.6 and 44.0, respectively), reflecting poorer HRQoL among STAART participants [63]. The mean SF-12 MCS score for STAART participants was $50.1(\mathrm{SD}=11.1)$; this is slightly lower than the average score for US men and women aged 60-69 (52.7 and 51.8, respectively), also reflecting somewhat poorer HRQoL among STAART participants [63]. We did not identify any comparator studies that included this measure.

\section{Duration of arthritis symptoms}

On average, the self-reported duration of arthritis symptoms was 13.1 years $(S D=10.0)$, with a longer duration for VA participants than UNC participants. In comparator studies, duration of symptoms ranged from 5 to 14 years, with most having a mean duration lower than the STAART study.

\section{Comorbid illnesses}

The mean number of self-reported comorbid illnesses among STAART participants was $8.5(\mathrm{SD}=3.9)$. Only two of the comparator studies reported a mean number of comorbidities for participants, and these were lower than in STAART (1.3-5.3), potentially indicating higher risk among our study sample $[57,59]$. However, because of different comorbidity measures, the ability to directly to compare studies is limited.

\section{BMI}

The mean BMI among STAART participants was $35.2 \mathrm{~kg} /$ $\mathrm{m}^{2}(\mathrm{SD}=8.2)$, which corresponds to Class 2 (moderate risk) obesity; BMI was somewhat lower in the VA group compared to the UNC group. In comparator studies, mean BMI's ranged from 30 to $34 \mathrm{~kg} / \mathrm{m}^{2}$, indicating more risk among STAART participants.

\section{Health behaviors \& coping strategies \\ Pain coping attempts - CSQ}

Among STAART participants, the mean Pain Coping Attempts score was 93.9 ( $\mathrm{SD}=36.6)$, with slightly higher scores for UNC participants. Two other studies reported 
this scale $[45,46]$, with scores ranging from 59 to 69; this indicates that STAART participants were overall engaged in a larger number of coping efforts compared to other studies.

\section{Pain catastrophizing - CSQ}

The mean Pain Catastrophizing Scale score was 11.4 $(\mathrm{SD}=7.6)$. This score was higher than two other studies reporting on this scale, in which mean scores ranged from 6.6-7.4 [25, 58]. This comparison suggests greater risk and needs for intervention among STAART participants.

\section{Pain catastrophizing scale}

The mean score on the PCS for STAART participants was $19.8(\mathrm{SD}=12.3)$. Among three other studies reporting on this scale, mean scores ranged from 7 to $17[45,55,59]$. This comparison suggests greater risk and needs for intervention among STAART participants.

\section{Depressive symptoms - PHQ-8}

In the STAART study, the mean PHQ-8 score was 6.2 $(\mathrm{SD}=5.3)$, and this was higher for VA participants than UNC participants. This average score indicates low depressive symptoms and is below the cutoff of 10 for depressive disorder [51]. We did not identify comparator studies that used this measure.

\section{Arthritis self efficacy scale}

The mean score for STAART participants on this measure was $5.9(\mathrm{SD}=2.0)$. Two other studies administered the same version of this scale, and the mean score was similar to STAART participants $[57,58]$.

\section{Self-efficacy for pain communication scale}

The mean score for STAART participants was 78.7 (SD $=22.0$ ); this score indicates a relatively low level of self-efficacy for communicating about pain [64]. We did not identify other comparator studies that used this measure.

\section{Brief fear of movement scale}

The mean score for STAART participants was 14.8 $(\mathrm{SD}=3.5)$ on a scale of $6-24$, suggesting a relatively high level of fear of movement. None of our comparator studies reported this measure.

\section{Discussion}

With a focus on health disparities, the STAART study aimed to reach a patient group with greater OA severity and risk for other negative OA-related outcomes. The study particularly focused on African Americans, who have reported worse OA-related symptoms compared to Caucasians in several studies $[2-4,65,66]$. We also selected recruitment sites that serve many patients with multiple health challenges and relatively low income levels, since these individuals may be at particular risk for worse OA-related outcomes. Based on descriptions of comparator studies, none had a particular focus on identifying patient populations with greatest risks or needs. We utilized proactive and culturally tailored recruitment methods [8] and were able to meet the study sample size goal within the specified timeline, potentially reflecting a high degree of receptivity to this type of intervention in this patient group. Consented patients were slightly younger than those who declined or were ineligible, but there was a more pronounced gender difference, with the consented group including more females than those who declined or were ineligible. This may be due to greater willingness of women to engage in behavioral and psychological interventions; additional work is needed to understand how to best engage men in these types of programs.

Comparisons to similar studies of pain CST and CBT-informed pain coping strategies demonstrate that STAART participants differ in a number of factors that reflect worse OA severity and greater vulnerability for worsening outcomes, across all three domains we examined within the NIMHD Research Framework:

\section{Sociocultural environment}

Based on our review of pain CST and or CBT-informed pain coping studies among patients with OA, STAART is the first to focus exclusively on African Americans. In most comparator studies, proportions of non-white participants were $10 \%$ or less, with none greater than about $1 / 3$. This emphasizes the uniqueness and importance of the STAART study in adding to understanding of pain CST interventions among African Americans with OA.

STAART participants also differ from prior studies demographically in other ways that may affect pain-related outcomes and response to a pain CST intervention. Fewer STAART participants are married or living with a partner as married, compared with other studies, likely reflecting the lower rates of marriage among African Americans than Caucasians in general [67]; however, these rates may also partly reflect a lower income status among STAART participants, considering the clinics in which we recruited and the fact that marriage rates decline with lower income. This is an important difference from other studies, since close partners can offer support for pain coping, and being "un-partnered" can place individuals at greater risk for to other health-related or psychosocial stressors $[23,46]$. To accommodate both married and single individuals and to reflect that pain communication goes beyond immediate support persons, the intervention encouraged participants to learn skills for communicating about pain with others more broadly, including family members, friends and health care providers. STAART participants, on average, 
are younger than samples of most prior studies in this area. This is likely due in part to higher risk of OA at younger ages among Veterans, who make up half of the STAART sample [68]. Although younger age is not necessarily a risk factor for worse pain-related outcomes, younger individuals with OA may be more likely to face challenges with continuing employment, particularly in physically demanding occupations. The STAART intervention is telephone-based, with a flexible schedule for calls, which may promote feasibility in working-age participants.

Although we were not able to directly compare financial status of participants across studies due to measurement inconsistency, about $1 / 3$ of STAART participants perceived they "just meet" or "don't even have enough to meet" basic expenses; as noted above, this may be partly reflect the underlying demographic characteristics of the clinics in which we recruited patients. This is important because financial stressors can augment the challenges of coping with chronic illnesses, and therefore individuals with lower income levels may particularly benefit from programs that teach and support coping skills. The telephone-based approach of STAART was also selected because it mitigates financial burdens related to transportation and missing work.

STAART participants reported relatively high levels of religiosity, which is important given the close connections between religiosity and multiple aspects of the pain and pain coping experience [69]. Unfortunately, we did not identify any comparator studies that measured this important construct. We expected that religious values would be important to a substantial proportion of STAART participants, and therefore one aspect of cultural tailoring involved encouraging participants to integrate elements of their spirituality or religious faith into their practice of pain coping when they felt it was important do so [8]. For example, during cognitive restructuring sessions, if participants identified that their faith played an integral role in reframing their pain-related challenges, this was further explored and built upon during the intervention.

\section{Biological vulnerability \& mechanisms}

Several key variables in this domain also indicate that STAART participants have greater risks and challenges than samples of comparator studies. First, the STAART sample overall had worse OA pain and function than participants in comparator studies. Although we could only make a direct comparison to studies that reported the same version of the WOMAC $[45,55,61]$, indirect comparisons to studies using other versions of the WOMAC, as well as the AIMS, suggest STAART participants had worse symptoms [25, 46, 57-59, 62]. The mean total WOMAC score for the STAART sample reflects moderate-to-severe symptoms. We expect this difference from other studies reflects worse OA symptoms among African Americans than Caucasians, which has been shown in a number of prior studies [2-4].

The STAART sample also had longer symptom duration than most comparator studies [55, 59, 61]. Although it is not clear whether the effectiveness of cognitive behavioral interventions differs based on time since symptom onset, it is an important consideration that overall, this group of patients had been managing their chronic pain for a longer period of time than patients in prior studies of this type. This difference from other studies may partly reflect a younger age of onset of OA among some military personnel and Veterans [68]. BMI was higher in our study than in any of the comparators, including Somers et al. [25], which selected only overweight and obese individuals. This is likely a reflection of higher BMI among African Americans in the US, compared with Caucasians [70]. Although it was difficult to compare comorbidity burden to other studies, STAART participants had a high number of comorbid illnesses (mean of 8.5). This likely reflects the high prevalence of multiple chronic health conditions (e.g. hypertension, diabetes, cardiovascular disease) among African Americans [70], but as noted above, this also may be partly attributable to the general patient populations of the clinics in which we recruited. Little is known about the associations of comorbidity with pain coping in OA, but the challenge of managing multiple health conditions may increase the difficulty of coping with OA-related symptoms.

\section{Health behaviors \& coping strategies}

STAART participants also differed from prior studies in ways that may indicate greater need for a pain CST intervention. First STAART participants reported higher levels of pain catastrophizing than any other study $[25,45,46,55,58,59]$; prior research has also found higher levels of catastrophizing among African Americans than Caucasians [11-13]. Pain catastrophizing can be improved by CST interventions, which emphasize cognitive restructuring as a strategy to address unhelpful thoughts about pain [20, 22, 24]. Participants in the STAART study also reported more coping attempts than other studies that measured this same construct $[45,46]$. This may be due in part to the higher levels of pain experienced by STAART participants compared with prior study samples.

STAART participants reported relatively low levels of Self-Efficacy for Pain Communication. Although none of our comparator studies measured this construct, STAART participants' scores were similar to those of another sample of individuals with OA [64]. Based on prior work by Campbell et al. [71], we expected that many patients would benefit from building skills and confidence in communicating with others about their pain experience; 
therefore, a pain communication module was included [8]. Fear of movement scores also were high among STAART participants. Although the STAART CST intervention does not specifically address fear of movement, other modules (e.g. activity pacing, cognitive restructuring) involve related concepts and have potential to reduce pain-related fear of movement. STAART participants had relatively low depressive symptoms, based on the mean PHQ-8 score. Although none of the comparator measures used this same measures, some studies measured depressive symptoms with other measures, including the Beck Depression Inventory, Depression Anxiety Stress Scales, Hospital Anxiety and Depression Scale, and the Geriatric Depression Scale [55, 58-61]; participants in these studies also had scores that indicated normal or low levels of depressive symptoms, similar to the STAART study.

\section{Conclusion}

In conclusion, this comparison of STAART participants to prior studies of CST for OA identified differences in a number of key variables related to OA severity and risks for poor pain-related outcomes. In particular, STAART participants have worse OA symptoms, higher BMI, and greater levels of pain catastrophizing compared to other study samples. STAART participants also have a high comorbidity burden, and $1 / 3$ perceive they have relatively low income. These characteristics place STAART participants at greater risk for worse OA-related physical and psychological outcomes. However, pain CST programs can improve multiple OA-related outcomes, and STAART participants may gain particular benefit from this intervention approach because of its focus on pain catastrophizing. If results of the STAART study support the effectiveness of pain CST in this group, this will be an important addition to prior literature, given the importance of identifying effective interventions for African Americans, who bear a higher burden of OA.

\section{Additional file}

Additional file 1: Participant Inclusion criteria and recruitment methods for comparator studies. (DOCX $26 \mathrm{~kb}$ )

\section{Abbreviations}

AIMS: Arthritis impact measurement scales; AIMS2-SF: AIMS2 Short Form: BMI: Body mass index; BWM: Behavioral weight management; CBT: Cognitive behavioral therapy; CSQ: Coping strategies questionnaire; CST: Coping skills training; DSM-IV: Diagnostic and statistics manual fourth edition; DUREL: Duke University Religion Index; HRQoL: Health-Related Quality of Life; MCS: Mental health composite score; NIMHD: National Institute on Minority Health and Health Disparities; OA: Osteoarthritis; PCS: Pain catastrophizing scale; PCS: Physical health composite score; PCST: Pain coping skills training PHQ-8: Patient Health Questionnaire 8; SD: Standard deviation; SF-12: ShortForm-12; STAART: Pain Coping Skills Training for African Americans with OsteoaRTthritis; UNC: University of North Carolina at Chapel Hill; VA: Durham VA Healthcare System; VAS: Visual analog scale; WOMAC: Western Ontario and McMasters Universities Osteoarthritis Index

\section{Acknowledgements}

The study team thanks all of the study participants, without whom this work would not be possible. We thank the following team members for their contributions to the research: Caroline Nagle, Kimberlea Grimm, Ashley Gwyn, Bernadette Benas, Alex Gunn, Leah Schrubbe, Catherine Stanwyck, Erin Haley and Scott Ravyts. The study team also expresses gratitude to the Stakeholder Panel for this project: Ms. Mae Karim, Ms. Sandy Walker, LPN (Chapel Hill Children's Clinic), Mr. Ralph Brown, Yashika Watkins, PhD, MPH (Movement is Life / University of Illinois at Chicago), Teresa J. Brady, PhD (Centers for Disease Control and Prevention), Elaine Hart-Brothers, MD, MPH (Community Health Coalition), and Ms. Laura C. Marrow (Arthritis Foundation). The study team also thanks all of the participants taking part in this research. We thank the authors of the comparator studies included in this manuscript, for their responsiveness regarding their study data: Kim L. Bennell et al., Christine Rini et al., Joan E. Broderick et al., Eeva-Eerika Helminen et al., Michael V. Vitiello et al., and M.V. Hurley et al.

\section{Funding}

Research reported in this manuscript was funded through a Patient-Centered Outcomes Research Institute (PCORI) Award (AD-1408-19519). The statements presented in this manuscript are solely the responsibility of the authors and do not necessarily represent the views of the Patient-Centered Outcomes Research Institute (PCORI), its Board of Governors, or Methodology Committee. KDA and LA receive support from National Institute of Arthritis and Musculoskeletal and Skin Diseases Multidisciplinary Clinical Research Center P60 AR062760. KDA and CJC receive support from the Center for Health Services Research in Primary Care, Durham VA Health Care System (CIN 13-410).

\section{Availability of data and materials}

The datasets generated and/or analyzed during the current study are not publicly available due to feasibility but are available (in de-identified form) from the corresponding author on reasonable request.

\section{Authors' contributions}

KDA, LCC, CJC, CWC, FJK, EZO, and TJS contributed to the study design. CJC and LA contributed to plans for and conduct of statistical analyses. LCC, TJS, and EH contributed to the development and administration of the pain coping skills training intervention. All authors contributed to drafting and editing of the manuscript. All authors approved the final manuscript.

\section{Ethics approval and consent to participate}

This research is in compliance with the Helsinki Declaration and was approved by the Institutional Review Boards of the University of North Carolina at Chapel Hill, Durham Veterans Affairs Medical Center, East Carolina University, and Duke University Health System. All study participants provided written informed consent.

\section{Consent for publication}

Not applicable

\section{Competing interests}

The authors declare that they have no competing interests.

\section{Publisher's Note}

Springer Nature remains neutral with regard to jurisdictional claims in published maps and institutional affiliations.

\section{Author details}

${ }^{1}$ Thurston Arthritis Research Center, University of North Carolina at Chapel Hill, 3300 Thurston Bldg., CB\# 7280, Chapel Hill, NC 27599-7280, USA. ${ }^{2}$ Department of Medicine, University of North Carolina at Chapel Hill, 125 MacNider Hall CB\# 7005, Chapel Hill, NC 27599, USA. ${ }^{3}$ Health Services Research and Development Service, Durham VA Medical Center, Durham, NC, USA. ${ }^{4}$ Department of Biostatistics and Bioinformatics, Duke University Medical Center, Durham, NC, USA. ${ }^{5}$ Department of Psychology, East Carolina University, Greenville, NC, USA. ${ }^{6}$ Department of Psychiatry and Behavioral Science, Duke University, Durham, NC, USA. ${ }^{7}$ Department of Medicine, Duke University Medical Center, Durham, NC, USA. ${ }^{8}$ Institute for Health Research and Policy, University of Illinois at Chicago, Chicago, IL, USA. 


\section{Received: 18 April 2018 Accepted: 27 August 2018}

\section{Published online: 19 September 2018}

\section{References}

1. Smith DM, Parmelee PA. Within-day variability of fatigue and pain among African Americans and non-Hispanic whites with osteoarthritis of the knee. Arthritis Care Res (Hoboken). 2016;68(1):115-22.

2. Allen KD. Racial and ethnic disparities in osteoarthritis phenotypes. Curr Opin Rheumatol. 2010;22(5):528-32.

3. Allen KD, Helmick CG, Schwartz TA, DeVellis B, Renner JB, Jordan JM. Racial differences in self-reported pain and function among individuals with radiographic hip and knee osteoarthritis: the Johnston County osteoarthritis project. Osteoarthritis Cartilage. 2009;17(9):1132-6.

4. Allen KD, Oddone EZ, Coffman CJ, Keefe FJ, Lindquist JH, Bosworth HB. Racial differences in osteoarthritis pain and function: potential explanatory factors. Osteoarthritis Cartilage. 2010;18:160-7.

5. Lavernia CJ, Alcerro JC, Rossi MD. Fear in arthroplasty surgery: the role of race. Clin Orthop Relat Res. 2010;468(2):547-54

6. Vina ER, Ran D, Ashbeck EL, Kwoh CK. Natural history of pain and disability among African-Americans and whites with or at risk for knee osteoarthritis: a longitudinal study. Osteoarthr Cartil. 2018;26(4):471-9.

7. IOM (Institute of Medicine). Initial National Piorities for Comparative Effectiveness Research. Washington DC: The National Academies Press; 2009.

8. Schrubbe LA, Ravyts SG, Benas BC, Campbell LC, Cene CW, Coffman CJ, Gunn AH, Keefe FJ, Nagle CT, Oddone EZ, et al. Pain coping skills training for African Americans with osteoarthritis (STAART): study protocol of a randomized controlled trial. BMC Musculoskelet Disord. 2016;17(1):359.

9. Meints SM, Stout M, Abplanalp S, Hirsh AT. Pain-related rumination, but not magnification or helplessness, mediates race and sex differences in experimental pain. J Pain. 2017:18(3):332-9.

10. Meints SM, Miller MM, Hirsh AT. Differences in pain coping between black and white Americans: a meta-analysis. J Pain. 2016;17(6):642-53.

11. Chibnall JT, Tait RC. Long-term adjustment to work-related low back pain: associations with socio-demographics, claim processes, and post-settlement adjustment. Pain Med. 2009;10(8):1378-88.

12. Edwards RR, Moric M, Husfeldt B, Buvanendran A, Ivankovich O. Ethnic similarities and differences in the chronic pain experience: a comparison of african american, Hispanic, and white patients. Pain Med. 2005;6(1):88-98.

13. Ruehlman LS, Karoly P, Newton C. Comparing the experiential and psychosocial dimensions of chronic pain in african americans and Caucasians: findings from a national community sample. Pain Med. 2005;6(1):49-60.

14. Tan G, Jensen MP, Thornby J, Anderson KO. Ethnicity, control appraisal, coping, and adjustment to chronic pain among black and white Americans. Pain Med. 2005;6(1):18-28.

15. Jones AC, Kwoh CK, Groeneveld PW, Mor M, Geng M, Ibrahim SA Investigating racial differences in coping with chronic osteoarthritis pain. J Cross Cult Gerontol. 2008;23:339-47.

16. Allen KD, Golightly YM, Olsen MK. Pilot study of pain and coping among patients with osteoarthritis: a daily diary analysis. J Clin Rheumatol. 2006; 12(3):118-23.

17. Somers TJ, Keefe FJ, Pells JJ, Dixon KE, Waters SJ, Riordan PA, Blumenthal JA, McKee DC, Lacaille L, Tucker JM, et al. Pain catastarophizing and painrelated fear in osteoarthritis patients: relationships to pain and disability. J Pain Symptom Manag. 2009;37(5):863-72

18. Somers TJ, Keefe FJ, Carson JW, Pells JJ, Lacaille L. Pain catastrophizing in borderline morbidly obese and morbidly obese individuals with osteoarthritic knee pain. Pain Res Manag. 2008;13(5):401-6.

19. Allen KD, Coffman CJ, Golightly YM, Stechuchak KM, Keefe FJ. Daily pain variations among patients with hand, hip, and knee osteoarthritis. Osteoarthritis Cartilage. 2009;17(10):1275-82.

20. Keefe FJ, Caldwell D, Williams DA, Gil KM, Mitchell D, Robertson C, Martinez S, Nunley J, Beckham JC, Helms M. Pain coping skills training in the management of osteoarthritic knee pain-ll: follow-up results. Behav Ther. 1990;21(4):435-47

21. Keefe FJ, Caldwell DS. Cognitive behavioral control of arthritis pain. Adv Rheumatol. 1997;81(1):277-90.

22. Keefe FJ, Caldwell DS, Baucom D, Salley A, Robinson E, Timmons K, Beaupre P, Weisberg J, Helms M. Spouse-assisted coping skills training in the management of knee pain in osteoarthritis: long-term followup results. Arthritis Care Res. 1999;12(2):101-11.
23. Keefe FJ, J.A. B, Baucom D, Affleck G, Waugh R, Caldwell DS, Beaupre P, Kashikar-Zuck S, Wright K, Egert J et al: Effects of spouse-assisted coping skills training and exercise training in patients with osteoarthritic knee pain: a randomized controlled study. Pain 2004, 110:539-549.

24. Riddle DL, Keefe FJ, Nay WT, McKee D, Attarian DE, Jensen MP. Pain coping skills training for patients with elevated pain catastrophizing who are scheduled for knee arthroplasty: a quasi-experimental study. Arch Phys Med Rehabil. 2011:92(6):859-65.

25. Somers TJ, Blumenthal JA, Guilak F, Kraus VB, Schmitt DO, Babyak MA, Craighead LW, Caldwell DS, Rice JR, MCKee DC, et al. Pain coping skills training and lifestyle behavioral weight management in patients with knee osteoarthritis: a randomized controlled study. Pain. 2012;153(6):1199-209.

26. Miranda J, Bernal G, Lau A, Kohn L, Hwang W-C. State of the science on psychosocial interventions for ethnic minorities. Annu Rev Clin Psychol. 2005;1:113-42.

27. Sullivan MJL, Thorn B, Haythornthwaite JA, Keefe FJ, Martin MI, Bradley LA, Lefebvre JC. Theoretical perspectives on the relation between catastrophizing and pain. Clin J Pain. 2001;17(1):52-64.

28. Allen KD, Bosworth HB, Coffman CJ, Lindquist JH, Sperber N, Weinberger M, Oddone EZ. Racial differences in pain coping efficacy in patients with hip and knee osteoarthritis. In: Annual meeting of the American College of Rheumatology: 2012; Washington, DC; 2012.

29. NIMHD Minority Health and Health Disparities Research Framework. https://www. nimhd.nih.gov/images/research-framework-slide.pdf. Accessed 4 Jan 2018.

30. Campbell LC, Keefe FJ, Scipio C, McKee DC, Edwards CL, Herman SH, Johnson LE, Colvin OM, McBride CM, Donatucci C. Facilitating research participation and improving quality of life for African American prostate cancer survivors and their intimate partners: a pilot study of telephonebased coping skills training. Cancer. 2006;109(2Suppl):414-24.

31. Project I.M.P.A.C.T. - Increase Minority Participation and Awareness of Clinical Trials: http://impact.nmanet.org/wordpress/. Accessed 4 Jan 2018.

32. Koenig HG, Bussing A. The Duke University religion index (DUREL): a fiveitem measure for use in epidemiological studies. Religions. 2010;1:78-85.

33. Bellamy N. WOMAC: a 20-year experiential review of a patient-centered selfreported health status questionnaire. J Rheumatol. 2002;29(12):2473-6.

34. Bellamy N, Buchanan WW, Goldsmith CH, Campbell J, Stitt LW. Validation study of WOMAC: a health status instrument for measuring clinically important patient relevant outcomes to antirheumatic drug therapy in patients with osteoarthritis of the hip or knee. J Rheumatol. 1988;15:1833-40.

35. Tubach F, Ravaud P, Baron G, Falissard B, Logeart I, Bellamy N, Bombardier C, Felson DT, Hochberg MC, van der Heijde D, et al. Evaluation of clinically relevant states in patient reported outcomes in knee and hip osteoarthritis: the patient acceptable symptom state. Ann Rheum Dis. 2005;64(1):34-7.

36. Quintana JM, Escobar A, Bilbao A, Arostegui I, Lafuente I, Vidaurreta I. Responsiveness and clinically important differences in WOMAC and SF-36 after hip joint replacement. Osteoarthritis Cartilage. 2005;13:1076-83.

37. Meenan RF, Gertman PM, Mason JH. Measuring health status in arthritis. The arthritis impact measurement scales. Arthritis Rheumatism. 1980;32(2):146-52.

38. Meenan RF, Mason JH, Anderson JJ, Guccione AA, Kazis L. AIMS2: the content and properties of a revised and expanded arthritis impact measurement scales health status questionnaire. Arthritis Rheumatism. 1992;35(1):1-10.

39. Ren XS, Kazis L, Meenan RF. Short-form arthritis impact measurement scales 2: tests of reliability and validity among patients with osteoarthritis. Arthritis Care Res. 1999;12(3):163-71.

40. Amtmann D, Cook KF, Jensen MP, Chen WH, Choi S, Revicki D, Cella D, Rothrock N, Keefe F, Callahan L, et al. Development of a PROMIS item bank to measure pain interference. Pain. 2010;150(1):173-82.

41. Ware J Jr, Kosinski M, Keller SD. A 12-item short-form health survey: construction of scales and preliminary tests of reliability and validity. Med Care. 1996:34(3):220-33.

42. Sangha O, Stucki G, Liang MH, Fossel AH, Katz JN. The self-administered comorbidity questionnaire: a new method to assess comorbidity for clinical and health services research. Arthritis Rheumatism. 2003;49(2):156-63.

43. Rosenstiel AK, Keefe FJ. The use of coping strategies in chronic low back pain patients: relationship of patient characteristics and current adjustment. Pain. 1983;17:33-44.

44. Hastie BA, Riley JL, Fillingim RB. Ethnic differences in pain coping: factor structure of the coping strategies questionnaire and coping strategies questionnaire-revised. J Pain. 2004;5(6):304-16.

45. Bennell KL, Nelligan R, Dobson F, Rini C, Keefe F, Kasza J, French S, Bryant C, Dalwood A, Abbott JH, et al. Effectiveness of an internet-delivered exercise 
and pain-coping skills training intervention for persons with chronic knee pain: a randomized trial. Ann Intern Med. 2017;166(7):453-62.

46. Keefe FJ, Caldwell DS, Baucom D, Salley A, Robinson E, Timmons K, Beaupre P, Weisberg J, Helms M. Spouse-assisted coping skills training in the management of osteoarthritic knee pain. Arthritis Care Res. 1996;9(4):279-91.

47. Keefe FJ, Caldwell DS, Queen K, Gil KM, Martinez S, Crisson JE, Ogden W, Nunley J. Osteoarthritis knee pain: a behavioral analysis. Pain. 1987;28:309-21.

48. Keefe FJ, Caldwell DS, Queen KT, Gil KM, Martinez S, Crisson JE, Ogden W, Nunley J. Pain coping strategies in osteoarthritis patients. J Consult Clin Psychol. 1987;55:208-12.

49. Parker JC, Smarr KL, Buescher KL, Phillips LR, Frank RG, Beck NC, Anderson SK, Walker SE. Pain control and rational thinking. Implications for rheumatoid arthritis. Arthritis Rheum. 1989;32(8):984-90.

50. Sullivan MJL, Bishop SR, Pivik J. The pain catastrophizing scale: development and validation. Psychol Assess. 1995;7:524-32.

51. Kroenke K, Strine TW, Spitzer RL, Williams JB, Berry JT, Mokdad AH. The PHQ8 as a measure of current depression in the general population. J Affect Disord. 2009;114(1-3):163-73.

52. Brady TJ. Measures of self-efficacy: arthritis self-efficacy scale (ASES), arthritis self-efficacy Scale-8 item (ASES-8), Children's arthritis self-efficacy scale (CASE), chronic disease self-efficacy scale (CDSES), Parent's arthritis selfefficacy scale (PASE), and rheumatoid arthritis self-efficacy scale (RASE). Arthritis Care Res (Hoboken). 2011;63(Suppl 11):S473-85.

53. Porter LS, Keefe FJ, Wellington C, DeWilliams A. Pain communication in the context of osteoarthritis: patient and partner self-efficacy for pain communication and holding back from discussion of pain and arthritisrelated outcomes. Clin J Pain. 2008;24(8):662-8.

54. Shelby RA, Somers TJ, Keefe FJ, DeVellis BM, Patterson C, Renner JB, Jordan JM. Brief fear of movement scale for osteoarthritis. Arthritis Care Res. 2012; 64(6):862-71.

55. Bennell KL, Ahamed Y, Jull G, Bryant C, Hunt MA, Forbes AB, Kasza J, Akram M, Metcalf $B$, Harris A, et al. Physical therapist-delivered pain coping skills training and exercise for knee osteoarthritis: randomized controlled trial. Arthritis Care Res (Hoboken). 2016;68(5):590-602.

56. Bennell KL, Ahamed Y, Bryant C, Jull G, Hunt MA, Kenardy J, Forbes A, Harris A, Nicholas M, Metcalf B, et al. A physiotherapist-delivered integrated exercise and pain coping skills training intervention for individuals with knee osteoarthritis: a randomised controlled trial protocol. BMC Musculoskelet Disord. 2012;13:129.

57. Rini C, Porter LS, Somers TJ, McKee DC, DeVellis RF, Smith M, Winkel G, Ahern DK, Goldman R, Stiller JL, et al. Automated internet-based pain coping skills training to manage osteoarthritis pain: a randomized controlled trial. Pain. 2015;156(5):837-48.

58. Broderick JE, Keefe FJ, Bruckenthal P, Junghaenel DU, Schneider S, Schwartz JE, Kaell AT, Caldwell DS, McKee D, Reed S, et al. Nurse practitioners can effectively deliver pain coping skills training to osteoarthritis patients with chronic pain: a randomized, controlled trial. Pain. 2014;155(9):1743-54.

59. Helminen EE, Sinikallio SH, Valjakka AL, Vaisanen-Rouvali RH, Arokoski JP. Effectiveness of a cognitive-behavioural group intervention for knee osteoarthritis pain: a randomized controlled trial. Clin Rehabil. 2015; 29(9):868-81.

60. Vitiello MV, McCurry SM, Shortreed SM, Balderson BH, Baker LD, Keefe FJ, Rybarczyk BD, Von Korff M. Cognitive-behavioral treatment for comorbid insomnia and osteoarthritis pain in primary care: the lifestyles randomized controlled trial. J Am Geriatr Soc. 2013;61(6):947-56.

61. Hurley MV, Walsh NE, Mitchell HL, Pimm TJ, Patel A, Williamson E, Jones RH, Dieppe $P$, Reeves BC. Clinical effectiveness of a rehabilitation program integrating exercise, self-management, and active coping strategies for chronic knee pain: a cluster randomized trial. Arthritis Care Res. 2007;57(7):1211-9.

62. Keefe FJ, Caldwell DS, Williams DA, Gil KM, Mitchell D, Robertson C, Martinez $\mathrm{S}$, Nunley J, Beckham JC, Crisson JE, et al. Pain coping skills training in the management of osteoarthritic knee pain: a comparative study. Behav Ther. 1990;21:49-62

63. Hanmer J, Lawrence WF, Anderson JP, Kaplan RM, Fryback DG. Report of nationally representative values for the noninstitutionalized US adult population for 7 health-related quality-of-life scores. Med Decis Mak. 2006; 26(4):391-400.

64. Porter LS, Keefe FJ, Wellington C, de Williams A. Pain communication in the context of osteoarthritis: patient and partner self-efficacy for pain communication and holding back from discussion of pain and arthritisrelated concerns. Clin J Pain. 2008;24(8):662-8.
65. Dillon CF, Rasch EK, Gu Q, Hirsch R. Prevalence of knee osteoarthritis in the United States: arthritis data from the Third National Health and Nutrition Examination Survey 1991-1994. J Rheumatol. 2006;33:2271-9.

66. Jordan JM, Helmick CG, Renner JB, Luta G, Dragomir AD, Woodard J, Fang F, Schwartz TA, Abbate LM, Callahan LF, et al. Prevalence of knee symptoms and radiographic and symptomatic knee osteoarthritis in African Americans and Caucasians: the Johnston County osteoarthritis project. J Rheumatol. 2007:31(4):172-80.

67. Raley RK, Sweeney MM, Wondra D. The growing racial and ethnic divide in U.S. marriage patterns. Futur Child. 2015;25(2):89-109.

68. Cameron KL, Hsiao MS, Owens BD, Burks R, Svoboda SJ. Incidence of physician-diagnosed osteoarthritis among active duty United States military service members. Arthritis Rheum. 2011;63(10):2974-82.

69. Dedeli O, Kaptan G. Spirituality and religion in pain and pain management. Health Psychol Res. 2013;1(3):e29.

70. Cunningham TJ, Croft JB, Liu Y, Lu H, Eke PI, Giles WH. Vital signs: racial disparities in age-specific mortality among blacks or African Americans United States, 1999-2015. MMWR Morb Mortal Wkly Rep. 2017;66(17):444-56.

71. Campbell LC, Keefe FJ, Scipio C, McKee DC, Edwards CL, Herman SH, Johnson LE, Colvin OM, McBride CM, Donatucci C. Facilitating research participation and improving quality of life for African American prostate cancer survivors and their intimate partners. A pilot study of telephonebased coping skills training. Cancer. 2007;109(2 Suppl):414-24.

Ready to submit your research? Choose BMC and benefit from

- fast, convenient online submission

- thorough peer review by experienced researchers in your field

- rapid publication on acceptance

- support for research data, including large and complex data types

- gold Open Access which fosters wider collaboration and increased citations

- maximum visibility for your research: over $100 \mathrm{M}$ website views per year

At BMC, research is always in progress.

Learn more biomedcentral.com/submissions 